\title{
ANÁLISE FUNCIONAL DE FALAS INAPROPRIADAS EM UMA PESSOA COM DIAGNÓSTICO DE
} ESQUIZOFRENIA

\section{FUNCTIONAL ANALYSIS OF INAPPROPRIATE SPEECH IN A PERSON WITH SCHIZOPHRENIA DIAGNOSIS}

\author{
ROBERTA MAIA MARCON ${ }^{1}$ \\ ILMA A. GOULART DE SOUZA BRITTO² \\ PONTIFÍCIA UNIVERSIDADE CATÓLICA DE GOIÁS, BRASIL \\ RESUMO
}

Este estudo objetivou utilizar a análise funcional (experimental) para identificar as variáveis controladoras das vocalizações inapropriadas de uma pessoa do sexo feminino, com 27 anos e diagnóstico de esquizofrenia. A análise funcional envolveu a manipulação de eventos ambientais em três condições principais, a saber, condição atenção, condição sozinha e, também, condição controle. Tais condições foram alternadas em um delineamento de pesquisa de múltiplos elementos. Os resultados da análise funcional demonstraram que a atenção social exerceu forte controle sobre a frequência de emissão de vocalizações inapropriadas. Esses achados possibilitaram discutir a utilidade do conceito de operação motivadora para analisar funcionalmente as respostas verbais emitidas por pessoas com diagnóstico de esquizofrenia.

Palavras-chave: respostas verbais, esquizofrenia, metodologia de análise funcional, atenção social.

This study implemented a functional (experimental) analysis to identify the controlling variables of the inapropriate vocalizations in a 27 years old female, diagnosed with schizophrenia. The functional analysis involved the manipulation of environmental events on three conditions: attention condition, alone condition, and control condition, which were conducted according to a multiple-element design. The results of the functional analysis showed that social attention exercised strong control over the frequency of occurrence of inappropriate vocalizations. These results allowed to discuss the usefulness of the concept of motivating operation to functionally analyze the verbal responses of persons with schizophrenia.

Keywords: verbal responses, schizophrenia, functional analysis methodology, social attention.

1E-mail: robertamarconpsi@gmail.com

2E-mail: psyilma@terra.com.br 
O modelo causal proposto por B. F. Skinner tem relações com a filosofia de $E$. Mach, um físico e epistemólogo do século XIX, e também com a teoria da seleção natural das espécies, de C. Darwin. Como o comportamento muda em função das interações do organismo com seu ambiente, Skinner (1938, 1953/2007) usou o termo análise funcional para referir-se à demonstração empírica das relações funcionais entre ambiente e comportamento, portanto, o efeito de uma variável (ambiental) sobre a outra (comportamento).

Para Skinner (1953/2007), qualquer condição ou evento que tenha efeito sobre o comportamento deve ser considerado. Quando se investiga a relação funcional entre essas condições e o comportamento, por meio da manipulação de variáveis controladoras, torna-se possível sua previsão e controle. Assim sendo, o uso da expressão "análise funcional" na abordagem skinneriana (i) especifica o comportamento como uma variável dependente, propondo-se a descrevê-lo em termos de condições físicas observáveis (direta e/ou indiretamente), e também (ii) especifica variáveis ambientais manipuláveis (variáveis independentes), com foco nos eventos antecedentes e consequentes.

Com essa forte fundamentação empírica, a abordagem analítico-comportamental considera que o comportamento deve ser analisado em termos das respostas controladas por estímulos antecedentes e por estímulos consequentes. Assim sendo, é tarefa dos analistas do comportamento buscar os eventos antecedentes e consequentes que controlam ou mantêm o comportamento, seja ele um comportamento adequado ou não. Tal fundamentação, aplicada em uma faixa ampla de possibilidades, é utilizada para explicar como os padrões de comportamento são selecionados, mantidos e fortalecidos por eventos antecedentes e consequentes (Chiesa, 1994/2006).

Iwata, Dorsey, Slifer, Bauman e Richman (1982/1994) apresentaram um estudo, cujo procedimento incluia elementos da experimentação, a denominada metodologia de análise funcional (functional analysis methodology), e que envolvia observar e registar a ocorrência de comportamentos-problema em diferentes condições - condição de atenção, demanda, sozinho e controle. Em cada condição, avaliaram-se os efeitos de diferentes consequências para o aumento ou a diminuição na frequência do comportamento-alvo. Ou seja, cada condição avaliava o efeito do reforçamento positivo, do reforçamento negativo e do reforçamento automático (Britto, 2009; Martin \& Pear, 2007/2009; O'Neill et al., 1997; Thompson \& Iwata, 2005; Wacker, 2000). Neste sentido, eventos antecedentes e consequentes eram experimentalmente manipulados.

Iwata et al. (1982/1994) empregaram essas condições para identificar os possíveis reforçadores do comportamento autolesivo apresentado por participantes com atraso no desenvolvimento. $\mathrm{Na}$ condição definida como atenção, o reforçador social positivo era disponibilizado em forma de atenção social (e.g., falas 'Não faça isso, você vai se machucar') contingente à autolesão. $\mathrm{Na}$ condição denominada demanda, um possível controle por reforço negativo era avaliado pela apresentação de tarefas. Nessa condição, a tarefa era interrompida contingentemente à ocorrência do comportamento autolesivo. $\mathrm{Na}$ condição sozinho, o participante ficava na sala de terapia, desacompanhado, e sem acesso a brinquedos ou outros materiais. Essa condição permitia aos pesquisadores identificar se o comportamento autolesivo produzia consequências não sociais. Tal situação poderia sugerir que o comportamento-alvo era mantido por reforçamento automático. Na condição controle, reforçadores sociais em forma de atenção, brincadeira ou tangíveis eram disponibilizados, sem a apresentação de contingências levemente aversivas que poderiam evocar o comportamento-alvo. Essa condição tinha o objetivo de avaliar se o comportamento-alvo ocorreria em uma situação na qual brinquedos eram disponibilizados e atenção social era provida em abundância.

Em linhas gerais, o estudo de Iwata et al. (1982/1994) apresentou uma metodologia para identificar possíveis fontes de controle do comportamento autolesivo (reforçamento social positivo na forma de atenção, reforçamento negativo na forma de fuga de demandas e reforçamento automático, na forma de estimulação ambiental produzida diretamente pela resposta - ver de Barros \& Benvenuti [2011]). A metodologia de análise funcional, por sua vez, se mostrou eficaz na identificação de variáveis mantenedoras do comportamento-alvo e, desde então, tem sido amplamente empregada em pesquisas aplicadas para investigar os eventos antecedentes e consequentes que mantém comportamentos-problema, tais como as vocalizações inapropriadas de pessoas com diagnóstico de esquizofrenia (e.g., Britto, Rodrigues, Alves, \& Quinta, 2010; DeLeon, Arnold, Rodriguez-Catter, \& Uy, 2003; Dixon, Benedict, \& Larson, 2001; Lancaster et al., 2004; Santana, 2008; Wilder, Masuda, O’Connor, \& Baham, 2001).

Em linhas gerais, ao incorporar procedimentos experimentais para investigar o comportamento-problema socialmente relevante, tal como permitido pela metodologia de análise funcional, questões complexas sobre as ações humanas poderão ser respondidas, o que contribui para o aprimoramento da análise do comportamento aplicada.

Nos estudos supracitados, frequências maiores de vocalização inapropriada têm sido observadas nas condições atenção e demanda, o que indica que este comportamento-problema seria função das variáveis antecedentes e consequentes disponibilizadas nessas condições (Britto et al., 2010; DeLeon et al., 2003; Dixon et al., 2001; Lancaster et al., 2004; Santana, 2008; Wilder et al., 2001).

Do exposto, entende-se como importante elucidar empiricamente o papel da atenção social no comportamento verbal de pessoas com diagnóstico de esquizofrenia, o que pode ser testado por meio de uma condição experimental de atenção, bem como sob quais outras condições (demanda, sozinho, controle) as repostas verbais ocorrem em frequências maiores. Isso porque, ao invés de adotar a explicação de que as pessoas se engajam 
em comportamentos desorganizados ou comportamentos altamente perturbadores porque são portadoras de algum tipo de transtorno mental (Britto, 2012), investiga-se a relação funcional entre os comportamentos por elas apresentados e os eventos ambientais que os mantêm; a análise funcional é, pois, uma tentativa eficaz de buscar a relação funcional (Skinner, 1953/2007; Britto \& Marcon, submetido).

O presente estudo objetivou utilizar a análise funcional (experimental) para identificar as variáveis controladoras das respostas verbais de uma pessoa com diagnóstico de esquizofrenia, visando contribuir com dados empíricos acerca das variáveis controladoras do comportamento-problema em questão e se constituir em condição para realização de estudos similares. A análise funcional envolveu a manipulação de eventos ambientais em três condições principais: condição atenção, sozinha e controle, as quais foram alternadas em um delineamento de pesquisa de múltiplos elementos.

Cumpre salientar que a condição de atenção foi empregada para testar o efeito de diferentes tipos de atenção social sobre a resposta verbal, sendo a atenção disponibilizada em forma de contato visual direto, contato físico, comentário e aprovação social. Outra variação metodológica foi a de incluir a solicitação de uma atividade na condição sozinha para testar o efeito desta em um ambiente ausente de consequências sociais. A condição controle diferiu da descrita no experimento de Iwata et al. (1982/1994), uma vez que dentre a ampla gama de reforçadores disponibilizados em abundância (i.e., objetos, alimentos), a atenção foi disponibilizada em menor magnitude.

\section{MÉTODO}

\section{Participante}

Participou deste estudo uma pessoa do sexo feminino, 27 anos, solteira, primeiro grau incompleto, nível socioeconômico baixo, que havia sido diagnosticada com esquizofrenia aos 21 anos. Fazia tratamento especializado em um Centro de Atenção Psicossocial (CAPS) há cerca de três anos. Quando ingressou no CAPS, queixava-se de dores de cabeça e tristeza e afirmava ouvir vozes que a mandavam matar pessoas. No passado, foi internada em uma clínica psiquiátrica onde permaneceu por três meses. Constava em seu prontuário que a participante fez uso dos seguintes psicofármacos: haloperidol, clorpromazina, prometazina, lorazepam, amitriptilina, imipramina, nortriptilina, biperideno e diazepam. Durante a coleta de dados, a participante fazia uso de duas ampolas de decanoato de haloperidol administradas quinzenalmente.

O projeto de pesquisa foi aprovado pelo Comitê de Ética em Pesquisa da Pontifícia Universidade Católica de Goiás (Protocolo número 0079.0.000.168-09).

\section{Materiais e Ambiente}

No decorrer do estudo foi utilizada uma câmera filmadora DVD-R (Disco Digital de Vídeo), aparelho de televisão, computador, impressora, papel no formato A4, lápis, caneta, prancheta, Folhas de Registro de Observação, gravuras, objetos (revistas, pulseiras, um par de brincos, presilhas de cabelo, uma lixa de unha e um chaveiro) e alimentos (bombons e bolachas). Esses objetos e alimentos foram definidos como reforçadores para esta participante, após entrevista com membros da equipe multiprofissional que a assistia.

As sessões experimentais foram realizadas na casa da participante - em uma sala mobiliada com três tamboretes e um armário de cozinha.

\section{Procedimento}

Foi feito contato com um CAPS a fim de solicitar a autorização para a realização deste estudo e selecionar um paciente que se enquadrasse nos critérios de inclusão desta pesquisa, entre eles: (a) ter idade acima de 18 anos; (b) apresentar diagnóstico psiquiátrico de esquizofrenia que não estivesse relacionado a efeitos fisiológicos de uma substância (e.g., uma droga de abuso), ou a uma condição médica geral; (c) apresentar vocalizações inapropriadas; e (d) estar em tratamento em CAPS.

A instituição e a participante foram informadas sobre os objetivos e o procedimento a ser utilizado na pesquisa, assim como sobre a importância do registro das sessões em vídeo. A coleta de dados foi iniciada somente após assinado o Termo de Consentimento Livre e Esclarecido pela participante e por um membro responsável da instituição.

\section{Avaliação funcional por observação indireta}

Com a finalidade de obter dados sobre a história de vida da participante, seus comportamentos-problema, e eventos potencialmente reforçadores a ela, a pesquisadora recorreu a dados do prontuário disponibilizado pela instituição e realizou entrevistas com membros da equipe multiprofissional que a assistia, assim como com familiares (e.g., mãe, tias) e uma vizinha, sua "cuidadora".

\section{Análise funcional experimental}

A análise funcional foi baseada nos procedimentos de Iwata et al. (1982/1994) com três condições principais: condição atenção (A), condição sozinha (S) e condição controle (C). A condição atenção (A) incluiu quatro subcondições: (A1) atenção - contato visual direto; (A2) atenção - contato físico; (A3) atenção - comentário; (A4) atenção - aprovação social. A condição sozinha (S) incluiu duas subcondições: (S1) sozinha, sem demanda; (S2) sozinha, com demanda. A condição controle (C) não incluiu subcondições. Para fidedignidade dos dados, todas as sessões foram registradas em vídeo.

\section{Condição atenção}

$\mathrm{Na}$ condição atenção, a pesquisadora permanecia na sala por cinco minutos, sentada em frente à participante, interagindo verbalmente com ela em conversas livres. As subcondições de atenção (contato visual direto, contato físico e comentário) 
diferiram quanto à forma de atenção disponibilizada, como especificado adiante.

\section{Condição atenção - contato visual direto (A1).}

A pesquisadora permanecia interagindo verbalmente com a participante, sem olhá-la diretamente enquanto falava com ela. A cada emissão de vocalização inapropriada, a pesquisadora, com expressão facial neutra e em silêncio, disponibilizava atenção à participante por cerca de 10 segundos, no formato de contato visual direto. Transcorrido esse tempo, voltava a falar com a participante.

\section{Condição atenção - contato físico (A2).}

A cada emissão de vocalização inapropriada, a pesquisadora disponibilizava atenção à participante por até 10 segundos, no formato de contato físico: tocava levemente, com uma de suas mãos, um dos joelhos da participante. Transcorrido esse tempo, voltava a interagir verbalmente com ela.

\section{Condição atenção - comentário (A3).}

A cada emissão de vocalização inapropriada, a pesquisadora inclinava o corpo em direção à participante $\mathrm{e}$ emitia o comentário: "Fica difícil compreender quando você fala assim".

\section{Condição atenção - aprovação social (A4).}

Para verificar se vocalizações inapropriadas ocorreriam quando da disponibilização de atenção a um comportamento apropriado alternativo (executar tarefa) e remoção de atenção ao comportamento-alvo, a pesquisadora mostrava uma gravura de uma revista à participante e solicitava que esta escrevesse um texto sobre a gravura apresentada. À medida que a participante executava a tarefa, a pesquisadora disponibilizava-lhe atenção social em forma de sinais de aprovação: verbalizava "Continue", "Mmm-hmm", ou movimentava a cabeça de cima para baixo ou emitia sorrisos. Quando da emissão de vocalização inapropriada, a pesquisadora não disponibilizava atenção social, mantendo-se com expressão facial neutra e em silêncio.

\section{Condição sozinha}

\section{Condição sozinha, sem demanda (S1).}

A pesquisadora solicitou à participante que a aguardasse por cinco minutos na sala. Logo após verbalizar esta orientação, a pesquisadora ausentou-se da sala, deixando a participante sozinha e a filmadora ligada.

Condição sozinha, com demanda (S2).

Para verificar se a vocalização inapropriada ocorreria sob controle da apresentação de uma tarefa na ausência de atenção social, a pesquisadora forneceu informações acerca de uma reportagem em uma revista e, logo após, solicitou à participante que produzisse um texto sobre a reportagem comentada. Em seguida, a pesquisadora ausentou-se da sala por cinco minutos, deixando a participante sozinha executando a atividade. A filmadora permaneceu ligada.

\section{Condição controle $(C)$.}

A pesquisadora colocou à disposição da participante objetos e alimentos definidos como reforçadores (revistas, pulseiras, um par de brincos, presilhas de cabelo, uma lixa de unha, um chaveiro, bombons e bolachas), os quais podiam ser livremente manuseados e/ou consumidos. A pesquisadora permaneceu na sala durante o tempo da sessão (cinco minutos), escrevendo em uma folha de papel. Na Tabela 1 consta o resumo de cada condição aplicada.

Tabela 1

Condições do Delineamento de Múltiplas Condições.

\begin{tabular}{|c|c|c|c|}
\hline Condição & Sessão & Duração & Procedimento \\
\hline Atenção contato visual direto & $1^{\mathrm{a}}$ & $5 \mathrm{~min}$ & $\begin{array}{l}\text { A fala inapropriada foi seguida por } 10 \text { segundos de } \\
\text { contato visual direto. }\end{array}$ \\
\hline Atenção contato físico & $2^{\mathrm{a}}$ & $5 \mathrm{~min}$ & $\begin{array}{l}\text { A fala inapropriada foi seguida por } 10 \text { segundos de } \\
\text { contato físico. }\end{array}$ \\
\hline Atenção comentário & $3^{\mathrm{a}}$ & $5 \mathrm{~min}$ & $\begin{array}{l}\text { A fala inapropriada foi seguida pelo comentário "Fica } \\
\text { difícil compreender quando você fala assim". }\end{array}$ \\
\hline Atenção aprovação social & $4^{\mathrm{a}}$ & $5 \mathrm{~min}$ & $\begin{array}{l}\text { A fala inapropriada não era consequenciada com } \\
\text { aprovação social, sendo esta disponibilizada para o } \\
\text { comportamento de executar tarefa. }\end{array}$ \\
\hline Sozinha sem demanda & $5^{\mathrm{a}}$ & $5 \mathrm{~min}$ & $\begin{array}{l}\text { A participante permaneceu na sala na ausência da } \\
\text { pesquisadora. }\end{array}$ \\
\hline Sozinha com demanda & $6^{\mathrm{a}}$ & $5 \mathrm{~min}$ & $\begin{array}{l}\text { A participante permaneceu na sala sob controle de uma } \\
\text { tarefa, na ausência da pesquisadora. }\end{array}$ \\
\hline Controle & $7^{\mathrm{a}}$ & $5 \mathrm{~min}$ & $\begin{array}{l}\text { A participante permaneceu na sala com reforçadores, } \\
\text { na presença da pesquisadora, que escrevia em uma } \\
\text { folha de papel. }\end{array}$ \\
\hline
\end{tabular}


As condições e subcondições experimentais foram aplicadas na seguinte sequência: A1, A2, A3, A4, $\mathrm{S} 1, \mathrm{~S} 2$ e C. Foi realizada a replicação dessas condições e subcondições, com exceção à condição C. Desse modo, quando da replicação das condições e subcondições, a seguinte sequência foi adotada: A1, A2, A3, A4, S1 e S2. Foram aplicadas duas condições e/ou subcondições por dia, portanto, duas sessões experimentais com duração de cinco minutos cada, transcorrendo um intervalo de 15 minutos entre uma e outra. As sessões foram conduzidas três vezes por semana, em um total de duas semanas. O intervalo de duas semanas correspondia ao intervalo de tempo que a dosagem da medicação era administrada na participante, variável a ser controlada, uma vez que o uso de medicação pode produzir efeito sobre o comportamento da pessoa em questão.

\section{Análise dos dados}

Após a realização do procedimento, foi iniciada a transcrição das respostas verbais registradas em vídeo. Foram definidas duas categorias principais de repostas verbais, assim denominadas: falas apropriadas (FA) e falas inapropriadas (FI). Definiu-se como FA, a emissão de palavras apropriadas ao contexto em questão (e.g., "Tem vez que eu choro. Já chorei muitas vezes assim, porque a mãe não tá"). As FI foram definidas como a emissão de palavras incomuns aos padrões convencionados pela comunidade verbal em questão, ou seja, verbalizações consideradas inapropriadas ao contexto em questão (e.g., a participante fazia referência à presença de réptil dentro do corpo, bem como ao controle de forças ocultas). Também foram consideradas FI, verbalizações repetitivas ou com elementos persecutórios, ou ameaçadores, ou místicos.

A partir dessa categorização foi realizada a contagem das FI e FA, separadamente. O registro da ocorrência e não ocorrência foi efetuado em uma Folha de Registro de Observação. Para obtenção da porcentagem das FI, multiplicou-se a quantidade total de FI emitidas em cada condição experimental por 100 . O resultado da multiplicação foi divido pelo total de FI emitidas durante todo o experimento. $\mathrm{O}$ mesmo foi feito em relação às $\mathrm{FA}$.

\section{Cálculo do Índice de Concordância}

Contou-se com a colaboração de duas pessoas graduadas com experiência em atividades que incluem observação e registro como observadores independentes para registrar a ocorrência e não ocorrência das FA e FI. O cálculo foi feito entre os pares de observadores: $\mathrm{AB}, \mathrm{BC}$ e $\mathrm{AC}$, sendo utilizada a fórmula: [Concordâncias / (concordâncias + discordâncias)] × 100 (Fagundes, 1999). $\mathrm{O}$ percentual calculado apontou altos índices de concordância para as FA (96\%) e para as FI (91\%). Esses percentuais referem-se à média das três concordâncias calculadas ( $\mathrm{AB}, \mathrm{BC}$ e $\mathrm{AC})$.

\section{RESULTADOS}

Na Figura 1 são apresentados os percentuais das FI que ocorreram nas aplicações e replicações das condições e subcondições experimentais empregadas neste estudo.

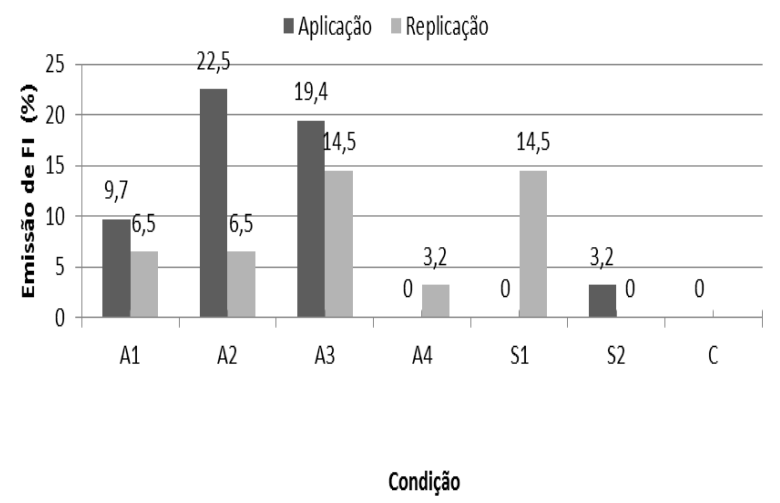

Figura 1. Porcentagem de emissão das falas inapropriadas nas diferentes condições.

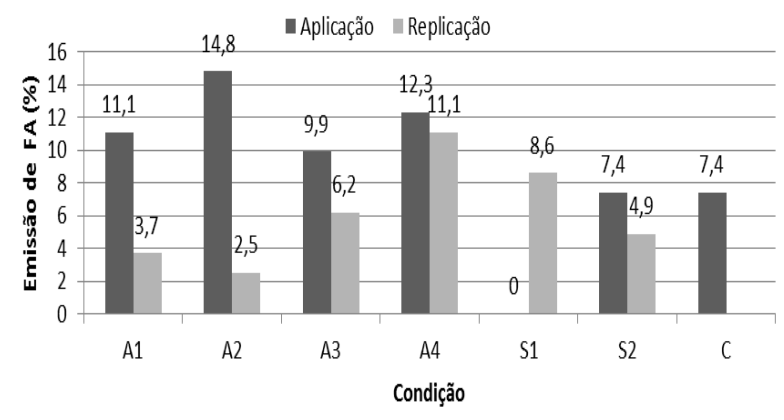

Figura 2. Porcentagem de emissão das falas apropriadas nas diferentes condições.

Conforme ilustrado na Figura 1, os maiores percentuais de FI - representativos do total das frequências de verbalizações obtidas minuto a minuto por sessão - durante a aplicação das condições e subcondições foram obtidos nas subcondições atenção contato físico (A2) - 22,5\% e atenção comentário (A3) - 19,4\%, seguidos pelas subcondições atenção contato visual direto (A1) - 9, 7\% e sozinha com demanda (S2) - 3,2\%. Percentual zero foi registrado para as subcondições atenção aprovação social (A4), sozinha sem demanda (S1) e condição controle $(C)$.

Em relação à replicação das subcondições, os maiores percentuais de FI foram obtidos nas subcondições atenção comentário (A3) e sozinha sem demanda (S1), com mesmo valor - $14,5 \%$, enquanto nas subcondições atenção contato visual direto (A1) e atenção contato físico (A2), registrou-se 6,5\%, para ambas. Para as subcondições atenção aprovação social (A4) e sozinha com demanda (S2), foram obtidos percentuais $3,2 \%$ e zero, respectivamente.

A partir dos achados apresentados na Figura 1, deve-se salientar que, somados os percentuais da aplicação e da replicação de cada uma das subcondições, o maior registro de emissão de FI deu-se na subcondição atenção comentário (A3) - 33,9\%, enquanto o menor percentual 
deste tipo de fala, nesta perspectiva, foi registrado nas subcondições atenção aprovação social (A4) e sozinha com demanda (S2) - 3,2\% em cada uma delas.

A Figura 2 apresenta os percentuais das FA que ocorreram nas aplicações e replicações das condições e subcondições empregadas neste estudo.

Conforme ilustrado na Figura 2, os maiores percentuais de FA - representativos do total das frequências de verbalizações obtidas minuto a minuto por sessão - durante a aplicação das condições e subcondições foram obtidos nas subcondições atenção contato físico (A2) $-14,8 \%$, atenção aprovação social (A4) - 12,3\% e atenção contato visual direto (A1) - 11,1\%, seguidos pelas subcondições atenção comentário (A3) - 9,9\%, sozinha com demanda (S2) e condição controle (C), ambas com o mesmo percentual $-7,4 \%$. Na subcondição sozinha sem demanda (S1), registrou-se percentual zero.

Quanto à replicação das subcondições, observouse que os maiores percentuais deste tipo de fala foram registrados nas subcondições atenção aprovação social (A4) - 11,1\%, sozinha sem demanda (S1) - 8,6\% e atenção comentário (A3) - 6,2\%, seguidos pelas subcondições sozinha com demanda (S2) - 4,9\%, atenção contato visual direto (A1) - 3,7\% e atenção contato físico (A2) $-2,5 \%$.

Desse modo, a partir dos achados apresentados na Figura 2, é possível ressaltar que somados os percentuais da aplicação e da replicação de cada uma das subcondições, o maior registro de emissão de FA deu-se na subcondição atenção aprovação social (A4) - 23,5\%, ao passo que o menor percentual deste tipo de fala, por esta perspectiva, deu-se na subcondição sozinha sem demanda (S1) $-8,6 \%$.

\section{DISCUSSÃO}

Os dados obtidos nas subcondições atenção contato visual direto (A1), atenção contato físico (A2) e atenção comentário (A3), possibilitam afirmar que a disponibilização de tipos específicos de atenção social contingente às FI (operação de reforçamento) e não disponibilização desse tipo de atenção social contingente às FA (suspensão da atenção) influenciaram a ocorrência das FI. Os resultados obtidos no presente estudo demonstraram maior frequência de FI em cada uma dessas subcondições de atenção, confirmando dados encontrados em estudos anteriores que empregaram essa metodologia de análise funcional (Britto et al., 2010; DeLeon et al., 2003; Dixon et al., 2001; Lancaster et al., 2004; Santana, 2008; Wilder et al., 2001). Uma exceção foi observada na replicação das condições, uma vez que a FI na condição sozinha sem demanda ocorreu com frequência similar à registrada em uma das subcondições de atenção (atenção comentário).

Esses dados ainda permitem constatar a utilidade do conceito de operação motivadora para a análise funcional da FI apresentada por pessoas com diagnóstico de esquizofrenia, uma vez que, nas condições de atenção, um tipo específico de atenção foi disponibilizado contingente à FI, enquanto a FA foi seguida pela suspensão da atenção, introduzindo uma operação motivadora que evocava FI controladas pela atenção (ver operações motivadoras e atenção social em Marcon \& Britto, 2011).

Importante salientar, acerca dos resultados obtidos na subcondição atenção comentário (A3), que a verbalização: "Fica difícil compreender quando você fala assim" contingente às FI reproduziu as verbalizações que comumente o contexto social de pessoas com o diagnóstico de esquizofrenia apresenta como consequência de suas FI. Portanto, o dado de que nessa subcondição a participante emitiu a maior frequência de FI é um dado que permite a seguinte generalização: verbalizações como "Não fala isso", "Pare de falar desse jeito", próprias do ambiente natural de pessoas com o diagnóstico de esquizofrenia - dado este observado por meio de fontes de informação por observação indireta, tal como entrevistas com pessoas significativas àquelas com diagnóstico de esquizofrenia -, disponibilizadas em consequência a essas falas inapropriadas, podem aumentar esse tipo de verbalização. Se assim for, esse tipo de consequência não deve ser apresentada por aqueles que atuam no contexto terapêutico e/ou por familiares dessas pessoas, posto que um efeito provável é o aumento na frequência desse tipo de verbalização (cf. Britto et al., 2010).

Por sua vez, na subcondição atenção aprovação social (A4), o procedimento se inverteu: a atenção passou a ser dispensada não mais contingente às FI, mas ao comportamento apropriado. Em decorrência da atenção social ter sido disponibilizada de forma contingente ao comportamento apropriado, as FA foram mais frequentes.

Em contrapartida, à medida que a pesquisadora se ausentava da sala, portanto, em ambas as subcondições da condição sozinha (S), a participante foi privada de atenção social. Assim, na condição sozinha (S) houve a presença de operação motivadora, uma vez que a atenção social estava suspensa, e ausência de reforçamento social positivo, apesar da possibilidade de reforçamento automático.

Um aspecto que parece contrariar os dados da literatura foi observado na condição sozinha (S), em cujas subcondições a participante emitiu FI e FA na ausência da pesquisadora, portanto, de atenção social. Necessário advertir que tanto os profissionais do CAPS quanto sua vizinha "cuidadora" afirmaram ser frequente ouvir a voz da participante, como se ela estivesse verbalizando com alguém que não estava presente. Há de se considerar que, em se tratando de uma pessoa com o diagnóstico de esquizofrenia, na condição sozinha ela não estaria, de fato, sozinha; ela é, pois, falante e ouvinte dela própria (Skinner, 1957/1978).

Em se tratando das subcondições atenção aprovação social (A4) e sozinha com demanda (S2), estas foram as que produziram menor percentual de ocorrência de FI. Esse dado corrobora a análise de que ao ocupar a participante com uma atividade estar-se-á favorecendo a ocorrência de comportamentos alternativos à ação verbal de apresentar FI.

$\mathrm{Na}$ condição controle (C), a participante teve acesso aos reforçadores disponibilizados. Assim, era ausente uma operação motivadora relacionada ao 
comportamento-problema. Um aspecto a ser observado em relação à condição controle (C) foi a não ocorrência de FI, tendo sido observadas apenas FA. Nessa condição, a participante estava sob controle de estímulos reforçadores que passaram a controlar suas falas apropriadas relacionadas, pois, a tais estímulos (e. g., "Linda as pulseirinhas", "Uma lixa, vou guardar", "Tão bonito esse brinco").

Cumpre salientar, ainda, que nesta condição, a participante apresentou sussurros, subcategorizados como falas inaudíveis, em maior frequência. Entretanto, este tipo de fala não foi classificado dentro das categorias destacadas - FI e FA -, ainda que se refira à resposta verbal. Isso pode ser justificado pelo tom de voz apresentado pela participante - inaudível - quando da ocorrência de sussurros, perante os quais o ouvinte não responde, por não serem audíveis os elementos que os compõem. Por tratar-se de uma classe de comportamento comum em pessoas diagnosticadas com esquizofrenia, é acertado concluir que este é um dado que merece ser mais bem estudado.

Destaca-se que conclusões válidas podem ser obtidas por meio de condições comumente limitadas a cinco minutos de duração e, também, por meio de uma (ou duas) repetições da aplicação da condição (cf., Martin \& Pear, 2007/2009). A repetição da aplicação da condição e/ou subcondições pode elucidar diferenças encontradas entre aplicação e replicação de uma mesma condição diferenças significativas no presente estudo foram encontradas na subcondição contato visual direto, contato físico e subcondição sozinha sem demanda. Todavia, o delineamento empregado não controlou um possível efeito da ordem de apresentação das condições, sendo adotada na aplicação a seguinte sequência A1, A2, A3, A4, S1, S2 e $\mathrm{C}$, também adotada na replicação, com exceção a $\mathrm{C}$. Em se tratando da replicação da condição controle, não foi possível uma nova aplicação dessa condição posto que os familiares da participante a levaram de volta a seu estado de origem, por solicitação do CAPS em que ela se tratava há três anos.

Esse aspecto, aliado à conveniência de aplicar todas as condições no mesmo dia parecem ser importantes. Nesse sentido, uma sugestão para replicação futura deste estudo é a de conduzir todas as condições, em sessões de cinco minutos, no mesmo dia, repetidas vezes, até que o comportamento-problema se apresente em estado estável. Assim, se poderia identificar sob qual (ou quais) condição a frequência do comportamento-problema é maior e a inferência de quais seriam as suas variáveis controladoras.

Sugere-se, ainda, como pesquisas futuras, a utilização da análise funcional que se refere à atividade experimental e, portanto, envolve a manipulação de variáveis para testar seus efeitos sobre o comportamento objeto de estudo. Isso porque a análise funcional possibilita maior eficácia na identificação de variáveis controladoras do comportamento e, assim, favorece o avanço de tecnologias comportamentais. Essa prática difere da atividade de identificar a relação funcional entre eventos comportamentais e ambientais a partir da coleta de informações por meio de entrevistas, observação, entre outras, que não incluem a manipulação de variáveis (Britto \& Marcon, submetido; Carr, 1997; Carr et al., 1994; Cone, 1997; Dunlap \& Kincaid, 2001; Martin \& Pear, 2007/2009).

Uma questão importante a ser pontuada diz respeito às dificuldades encontradas na realização de estudos desta natureza. Entre elas, a falta de assiduidade da participante, que se ausentava do CAPS antes do horário predefinido para as sessões, embora este tenha sido disponibilizado pela instituição, alegando não poder utilizar o transporte coletivo após aquele período. Desse modo, a pesquisa foi realizada na casa da participante.

\section{REFERÊNCIAS}

Britto, I. A. G. S. (2009). Esquizofrenia: Intervenções operantes. In R. C. Wielenska (Org.), Sobre comportamento e cognição: Desafios, soluções e questionamentos (Vol. 23, pp. 393-401). Santo André: ESETec.

Britto, I. A. G. S. (2012). Psicopatologia e Análise do Comportamento: Algumas reflexões. Boletim Contexto, 37(2), 54-76.

Britto, I. A. G. S., \& Marcon, R. M. (Submetido). Procedimentos de avaliação e análise funcional em contextos aplicados. Revista Brasileira de Análise do Comportamento.

Britto, I. A. G. S., Rodrigues, I. S., Alves, S. L., \& Quinta, T. L. S. S. (2010). Análise funcional de comportamentos verbais inapropriados de um esquizofrênico. Psicologia: Teoria e Pesquisa, 26(1), 67-72.

Carr, E. G. (1977). The motivation of self-injurious behavior: A review of some hypotheses. Psychological Bulletin, 84, 800-816.

Carr, E. G., Levin, L., McConnachie, G., Carlson, J. I., Smith, C. E., \& Kemp, D. C. (1994). Communicationbased intervention for problem behavior. Baltimore: Paul H. Brookes.

Cone, J. D. (1997). Issues in functional analysis in behavioral assessment. Behavior Research and Therapy, 35, 259-275.

Chiesa, M. (2006). Behaviorismo radical: A filosofia e a ciência. (Trad. C. E. Cameschi). Brasília: Editora Celeiro (Trabalho original publicado em 1994).

de Barros, T., \& Benvenuti, M. F. L. (2011). Reforçamento automático: estratégias de análise e intervenção. Acta Comportamentalia, 20(2), 177-184.

DeLeon, I. G., Arnold, K. L., Rodriguez-Catter, V., \& Uy, M. L. (2003). Covariation between bizarre and nonbizarre speech as a function of the content of verbal attention. Journal of Applied Behavior Analysis, 36(1), 101-104.

Dixon, M. R., Benedict, H., \& Larson, T. (2001). Functional analysis and treatment of inappropriate verbal behavior. Journal of Applied Behavior Analysis, 34(3), 361-363.

Dunlap, G., \& Kincaid, D. (2001). The widening world of functional assessment: Comments on four manuals and beyond. Journal of Applied Behavior Analysis, 34(3), 365-377. 
Fagundes, A. J. F. M. (1999). Descrição, definição e registro de comportamento (12 ${ }^{\mathrm{a}}$ Edição). São Paulo: EDICON.

Iwata, B. A., Dorsey, M. F., Slifer, K. J., Bauman, K. E., \& Richman, G. S. (1994). Toward a functional analysis of self-injury. Journal of Applied Behavior Analysis, 27(2), 197-209. (Reedição de Analysis and Intervention in Developmental Disabilities, 3, 3-20, 1982).

Lancaster, B. M., LeBlanc, L. A., Carr, J. E., Brenske, S., Peet, M. M., \& Culver, S. J. (2004). Functional analysis and treatment of the bizarre speech of dually diagnosed adults. Journal of Applied Behavior Analysis, 37(3), 395-399.

Marcon, R. M., \& Britto, I. A. G. S. (2011). Operações motivadoras e atenção social: Eventos relevantes para comportamentos-problema de esquizofrênicos. Revista Perspectivas em Análise do Comportamento, 2(2), 192-202.

Martin, G., \& Pear, J. (2009). Modificação de comportamento: O que é e como fazer. ( $8^{\mathrm{a}}$ ed.; N. C. Aguirre, Trad.). São Paulo: Roca. (Trabalho original publicado em 2007).

O’Neill, R. E., Horner, R. H., Albin, R. W., Sprague, J. R., Storey, K., \& Newton, J. S. (1997). Functional assessment and program development for problem behavior: A practical handbook. Pacific Grove: Brooks/Cole.

Santana, L. A. M. (2008). Comportamento verbal $e$ esquizofrenia: Estratégias operantes de intervenção.
Dissertação de Mestrado. Goiania, GO: Pontifícia Universidade Católica de Goiás.

Skinner, B. F. (1938). The concept of the reflex in the description of behavior. Journal of General Psychology, 5, 427- 458.

Skinner, B. F. (2007). Ciência e comportamento humano. (11 ${ }^{\mathrm{a}}$ ed.; J. C. Todorov \& R. Azzi, Trads.). São Paulo: Martins Fontes. (Trabalho original publicado em 1953).

Skinner, B. F. (1978). O comportamento verbal. Tradução organizada por M. P. Villalobos. São Paulo: Cultrix Editora da USP. (Trabalho original publicado em 1957).

Thompson, R. H., \& Iwata, B. A. (2005). A review of reinforcement control procedures. Journal of Applied Behavior Analysis, 38(2), 257-278.

Wacker, D. P. (2000). Building a bridge between research in experimental and applied behavior analysis. Em: J. C. Leslie \& D. Blackman (Orgs.), Experimental and applied analysis of human behavior (pp. 205-212). Reno: Context Press.

Wilder, D. A., Masuda, A., O'Connor, C., \& Baham, M. (2001). Brief functional analysis and treatment of bizarre vocalizations in an adult with schizophrenia. Journal of Applied Behavior Analysis, 34(1), 65-68.

Recebido em 02/11/2014

Aceite final em 27/03/2015

Editor Associado: Edson Massayuki Huziwara 